\title{
RADIO POLARIMETRY OF THE ELAIS N1 FIELD: POLARIZED COMPACT SOURCES
}

\author{
A. R. Taylor, ${ }^{1}$ J. M. Stil ${ }^{1}$ J. K. Grant, ${ }^{1}$ T. L. Landecker, ${ }^{2}$ R. Kothes, ${ }^{2}$ R. I. Reid, ${ }^{2}$ A. D. Gray, ${ }^{2}$ \\ Douglas Scott, ${ }^{3}$ P. G. Martin, ${ }^{4}$ A. I. Boothroyd, ${ }^{4}$ G. Joncas, ${ }^{5}$ Felix J. Lockman, ${ }^{6}$ \\ J. English, ${ }^{7}$ A. SaJina, ${ }^{8}$ and J. R. Bond ${ }^{9}$ \\ Received 2007 February 27; accepted 2007 May 11
}

\begin{abstract}
We present deep polarimetric observations at $1420 \mathrm{MHz}$ of the European Large Area ISO Survey North 1 region (ELAIS N1) as part of the Dominion Radio Astrophysical Observatory Planck Deep Fields project. By combining closely spaced aperture synthesis fields, we image a region of $7.43 \mathrm{deg}^{2}$ to a maximum sensitivity in Stokes $Q$ and $U$ of $78 \mu \mathrm{Jy}_{\text {beam }^{-1}}$, and detect 786 compact sources in Stokes $I$. Of these, 83 exhibit polarized emission. We find that the differential source counts $(\log N-\log p)$ for polarized sources are nearly constant down to $p>500 \mu \mathrm{Jy}$, and that these faint polarized radio sources are more highly polarized than the strong source population. The median fractional polarization is $4.8 \% \pm 0.7 \%$ for polarized sources with Stokes $I$ flux density between 10 and 30 mJy, approximately 3 times larger than sources with $I>100 \mathrm{mJy}$. The majority of the polarized sources have been identified with galaxies in the Spitzer Wide Area Infrared Extragalactic Survey (SWIRE) image of ELAIS N1. Most of the galaxies occupy regions in the IRAC $5.8 \mu \mathrm{m} / 3.6 \mu \mathrm{m}$ versus $8.0 \mu \mathrm{m} / 4.5 \mu \mathrm{m}$ color-color diagram associated with dusty AGNs, or with ellipticals with an aging stellar population. A few host galaxies have colors that suggests significant PAH emission in the near-infrared. A small fraction, 12\%, of the polarized sources are not detected in the SWIRE data. None of the polarized sources in our sample appears to be associated with an actively star-forming galaxy.
\end{abstract}

Subject headings: galaxies: evolution — galaxies: individual (ELAIS N1) — polarization radio continuum: galaxies - techniques: polarimetric

\section{INTRODUCTION}

Observation of polarized radiation at radio wavelengths is one of the prime means to study the roles of magnetic fields in astrophysics, through synchrotron emissivity that samples magnetic fields in relativistic plasmas and through Faraday rotation, created by radiation propagation through magnetized thermal plasmas. Understanding the origin and evolution of magnetic fields is a key science goal of the Square Kilometer Array (SKA), a nextgeneration radio telescope under development by the international community (Schilizzi 2004). The primary observational tool to study the magnetic universe will be an SKA all-sky rotation measure survey of background radio sources and diffuse Galactic emission down to polarized flux density levels of $\sim 0.1 \mu \mathrm{Jy}$ (Beck \& Gaensler 2004). However, while modern source counts approach flux density sensitivities of $\sim 10 \mu \mathrm{Jy}$ in total intensity (Windhorst 2003; Hopkins et al. 2003), very little is known about the polarization properties of the faint radio source population. The most extensive analyses of polarization of compact extragalactic sources were carried out by Mesa et al. (2002) and Tucci

\footnotetext{
1 Department of Physics and Astronomy, University of Calgary, Calgary, AB T2N 1N4, Canada.

2 Dominion Radio Astrophysical Observatory, Herzberg Institute of Astrophysics, National Research Council of Canada, Penticton, BC V2A 6J9, Canada.

3 Department of Physics and Astronomy, University of British Columbia, Vancouver, BC V6T 1Z1, Canada.

4 Department of Astronomy, University of Toronto, Toronto, ON M5S 3H4, Canada.

5 Département de physique, génie physique, et d'optique, Université Laval, Quebec, QC G1K 7P4, Canada.

${ }^{6}$ National Radio Astronomy Observatory, Green Bank, WV 24944.

7 Department of Physics, University of Manitoba, Winnipeg, MB R3T 2N2, Canada.

${ }^{8}$ Spitzer Science Center, California Institute of Technology, Pasadena, CA 91125.

9 Canadian Institute for Theoretical Astrophysics, University of Toronto, Toronto, ON M5S 3H8, Canada.
}

et al. (2004), who used the NVSS data (Condon et al. 1998) to derive statistical polarization properties for $\sim 30,000$ sources with $S_{1.4 \mathrm{GHz}}>100$ mJy. Similarly, Beck \& Gaensler (2004) used NVSS sources with total flux density greater than $80 \mathrm{mJy}$ to extrapolate polarized source counts to $\mu \mathrm{Jy}$ levels.

Mesa et al. (2002) found that the mean fractional polarization of radio sources in the NVSS brighter than $80 \mathrm{mJy}$ was anticorrelated with flux density, especially for steep-spectrum radio sources $\left(\alpha<-0.5\right.$, for $\left.S_{\nu} \sim \nu^{\alpha}\right)$. Tucci et al. (2004) confirmed this result for the median of the fractional polarization for steepspectrum sources only ( $87 \%$ of their sample), but found no significant trend for flat-spectrum sources (13\% of their sample). Tucci et al. (2004) also noted that the flat shape of the polarized source counts indicates a dependence of the fractional polarization on flux density.

Radio sources with $S_{1.4 \mathrm{GHz}}>100 \mathrm{mJy}$ are predominantly associated with flat- or steep-spectrum active galactic nuclei (AGNs). Star-forming galaxies begin to be a significant fraction of the population at flux densities less than a few millijanskys (see, e.g., Hopkins et al. 2000; Windhorst 2003). However, there is still ongoing debate about the fraction of the submillijansky radio sources that are radio-quiet AGNs (Gruppioni et al. 1999; Simpson et al. 2006). It is thus uncertain that polarization properties derived from the strong radio source population are applicable to the submillijansky radio sources.

We have begun a project called the DRAO Planck Deep Fields to explore the high-latitude sky at high sensitivity in polarized radio continuum and in atomic hydrogen emission. The project uses the DRAO Synthesis Telescope at $1.4 \mathrm{GHz}$ to create deep images of two fields, one with a very low column of foreground material, the ELAIS N1 region $(l, b)=\left(84^{\circ},+45^{\circ}\right)$, and a larger region of highly structured infrared cirrus emission at $(l, b)=$ $\left(135^{\circ},+40^{\circ}\right)$. This paper reports initial results from the first $30 \%$ of observations of ELAIS N1. The ELAIS N1 (European Large Area ISO Survey North 1) field (Oliver et al. 2000) is an area of 
approximately $2 \mathrm{deg}^{2}$ chosen for a mid-infrared survey of distant galaxies with $I S O$. The field was selected to minimize confusion with Galactic cirrus and the zodiacal background, being one of the lines of sight to the extragalactic sky with minimum IRAS $100 \mu \mathrm{m}$ emission. A larger area that includes ELAIS N1 was later observed by the Spitzer Wide Area Infrared Extragalactic Survey (SWIRE) (Lonsdale et al. 2003). The large amount of archival data from these extragalactic surveys make this region ideal for studies of the faint polarized radio source population.

\section{OBSERVATIONS AND DATA PROCESSING}

\subsection{Synthesis Observations}

The DRAO Synthesis Telescope (DRAO ST) is described in detail in Landecker et al. (2000). The telescope is a seven-element east-west array of $9 \mathrm{~m}$ diameter antennas. Three antennas are moved to provide complete sampling of the UV plane from the shortest baseline $(12.86 \mathrm{~m})$ to the longest baseline $(617.18 \mathrm{~m})$ after a full synthesis of $12 \times 12 \mathrm{hr}$. The array has a primary beam size $107.2^{\prime}$ (FWHM) at $1420 \mathrm{MHz}$, which makes it an effective instrument for wide-field surveys. The first sidelobe of the synthesized beam is at the $3 \%$ level, and sidelobes farther from the main lobe of the beam are less than $0.5 \%$. The first grating ring of the synthesized beam appears at $2.8^{\circ}$ from the main lobe at $1420 \mathrm{MHz}$, which is outside the field of view defined by the $10 \%$ sensitivity level of the primary beam.

The telescope observes simultaneously the $\mathrm{H}$ i $21 \mathrm{~cm}$ line and continuum at $408 \mathrm{MHz}$ and full polarimetry in four $7.5 \mathrm{MHz}$ wide frequency bands centered around $1420 \mathrm{MHz}$. The antennas have prime focus feeds and at $1420 \mathrm{MHz}$ receive both right-hand (R) and left-hand (L) circularly polarized radiation. The observations and data processing techniques used in this paper follow those that have been used to obtain high-fidelity wide-field polarimetric images of the Galactic plane with the DRAO ST (Taylor et al. 2003; T. L. Landecker et al. 2007, in preparation).

The system temperature of the telescope as described by Landecker et al. (2000) was $60 \mathrm{~K}$, leading to an rms noise in a synthesized image of a single field of $280 \mu \mathrm{Jy}_{\text {beam }}{ }^{-1}$ at field center. However, starting in 2003, the sensitivity was enhanced by a series of improvements, completed by the time the current observations began in 2004. These improvements comprised installation of new low-noise amplifiers, modifications to telescope structures to reduce ground noise, and installation of shielding fences to further block ground radiation from entering the aperture. The system temperature of the seven individual antennas now spans the range $35-60 \mathrm{~K}$, and the overall system temperature is $\sim 45 \mathrm{~K}$, leading to a field-center rms noise of $210 \mu \mathrm{Jy} \mathrm{beam}^{-1}(53 \sin \delta \mathrm{mK})$ at $1420 \mathrm{MHz}$. A higher sensitivity and larger field of view are obtained by creating a mosaic of overlapping fields. The most uniform sensitivity across a mosaic is obtained if the fields are centered on a hexagonal grid. The field-center separation for the survey presented here is $22^{\prime}$, which is $20.5 \%$ of the FWHM diameter of the primary beam.

Observations for the DRAO ELAIS N1 survey began in 2004 August and will continue to create a final mosaic of 30 fields. This paper presents $21 \mathrm{~cm}$ continuum polarimetry of the first 10 fields. Figure 1 shows the location of the 10 -field mosaic in relation to the ISO ELAIS N1 field, and the SWIRE survey of ELAIS N1. The theoretical maximum $1 \sigma$ sensitivity in our mosaic of 10 fields is $\sim 80 \mu \mathrm{Jy}$.

\subsection{Polarization Data Processing and Calibration}

Complex gains for the center of each field were determined by observing the unresolved and unpolarized sources 3C 147 and

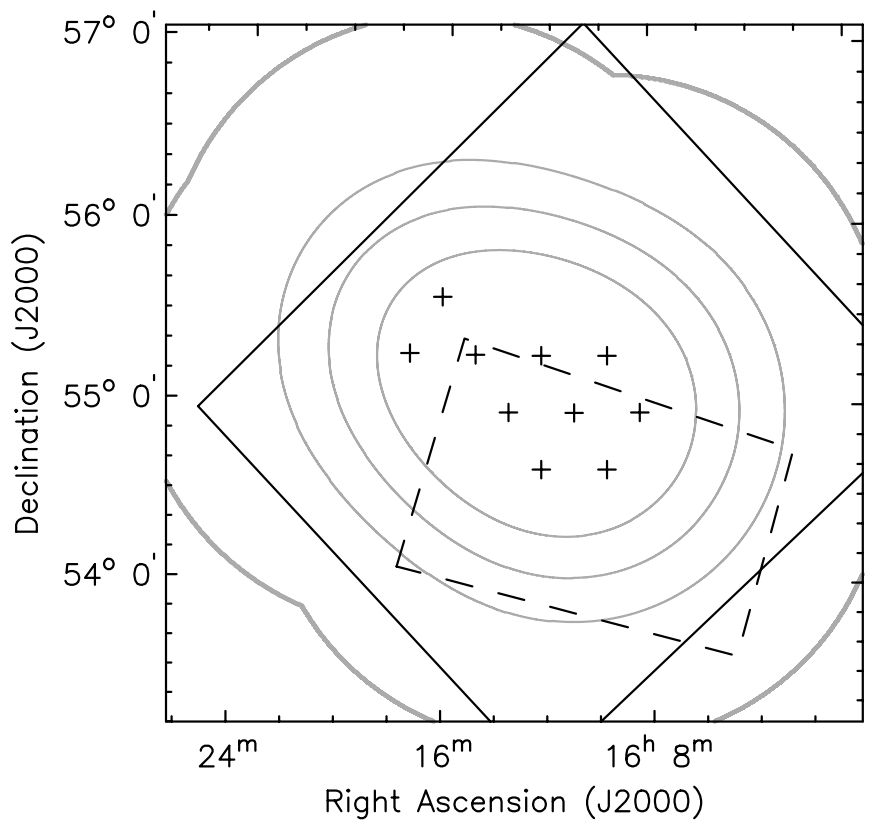

FIG. 1.- Survey area of the first 10 fields of the DRAO mosaic in relation to the sky coverage of the original ELAIS N1 field (dashed lines) and the SWIRE survey (solid black lines). The 10 field centers are indicated by the plus signs. Gray contours indicate the sensitivity at 1.5, 2, and 3 times the theoretical noise level in the center of the mosaic, and the edge of the field of view (thick line).

3C 295 between $12 \mathrm{hr}$ observing runs. The absolute polarization angle was calibrated by observing the highly linearly polarized source 3C 286 once every 4 days.

The polarization images do not contain much flux, making selfcalibration ineffective. Therefore $\mathrm{R}$ and $\mathrm{L}$ gain solutions derived from self-calibration of the Stokes $I$ images were applied to the polarization data as well. The resulting visibility data sets for each field were then corrected for the effects of instrumental polarization across the field of view (which leads to leakage of Stokes $I$ power into $Q$ and $U$ ). Instrumental polarization is a complex quantity, with phase and amplitude terms. These were measured for the seven individual antennas with a holographic technique, using the unpolarized source $3 \mathrm{C} 295$, on a $15^{\prime}$ grid across the primary beam. After interpolation, these measurements were used to predict conversion of $I$ into $Q$ and $U$ at any point in the beam based on CLEAN component models from processing the $I$ image for a field. Residual errors in instrumental polarization for an individual field are estimated at $0.25 \%$ for the field center, growing to $1 \%$ at a distance of $75^{\prime}$. With a field separation in the mosaic image of $22^{\prime}$ the instrumental polarization in the central $3.6 \mathrm{deg}^{2}$ of the mosaic remains less than $0.5 \%$. Toward the edge of the mosaic instrumental terms may be as large as $1 \%$.

After these initial procedures there are usually still confusing arclike structures left in the images, centered on bright sources inside and outside the primary beam. These are the result of residual complex gain errors at large distance from the field center. Effects from these are removed from the visibilities using a procedure called modcal, which is in principle a direction-dependent self-calibration (Willis 1999).

The antenna sidelobes are highly polarized, and sources outside the primary beam can produce strong spurious polarized signals. The Sun is seen in the sidelobes whenever it is above the horizon, but its effects are usually confined to the shorter baselines because of its large extent. The effects of the Sun were removed by making images centered on the Sun's position and removing the response from the visibilities. Terrestrial 

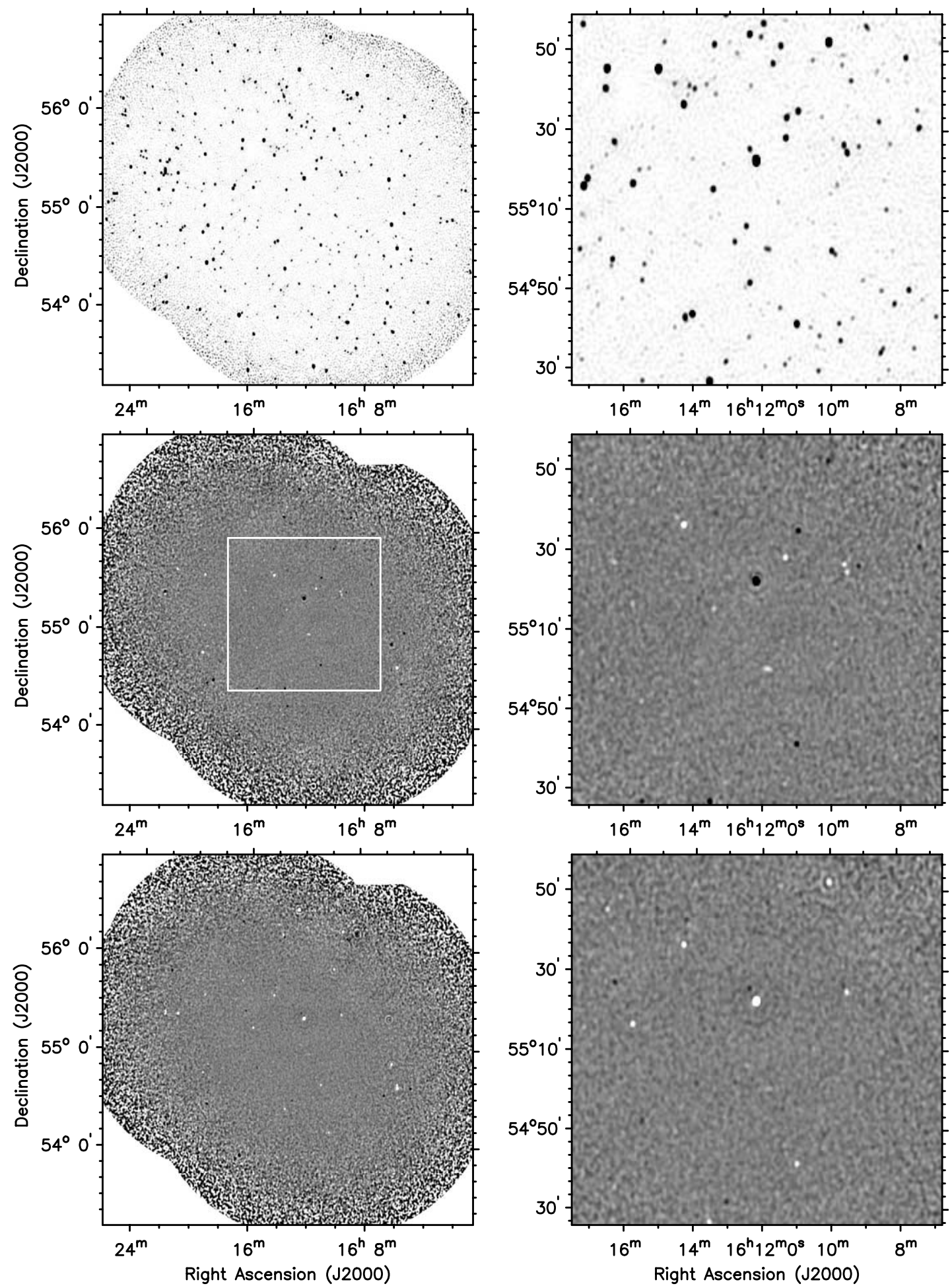

FIG. 2.-Continuum images of the ELAIS N1 field at $21 \mathrm{~cm}$ wavelength. Top panels: Stokes $I$ (gray scales linear from -0.1 to $+5 \mathrm{mJy}^{-1}$ beam ${ }^{-1}$ ). Middle panels: Stokes $Q$ (gray scales linear from -1 to $+1 \mathrm{mJy}$ beam ${ }^{-1}$ ). Bottom panels: Stokes $U$ (gray scales linear from -1 to $+1 \mathrm{mJy}$ beam ${ }^{-1}$ ). Panels on the right show an enlargement of the area indicated by the white frame in the Stokes $Q$ image.

interference, which is always polarized, is another source of spurious polarization. Interference effects in images appear as a spurious source concentrated around the north celestial pole, and can be largely eliminated by making an image at the pole and correcting the visibilities. Radiation from the ground appears to be polarized, and can appear as a correlated signal in visibilities corresponding to short baselines; this effect is more difficult to remove. In some particularly bad cases the data for affected interferometer spacings were simply flagged and removed.

\subsection{The Images}

Figure 2 shows the deep $21 \mathrm{~cm}$ continuum images of the ELAIS N1 area in Stokes $I, Q$, and $U$. The images are centered on 


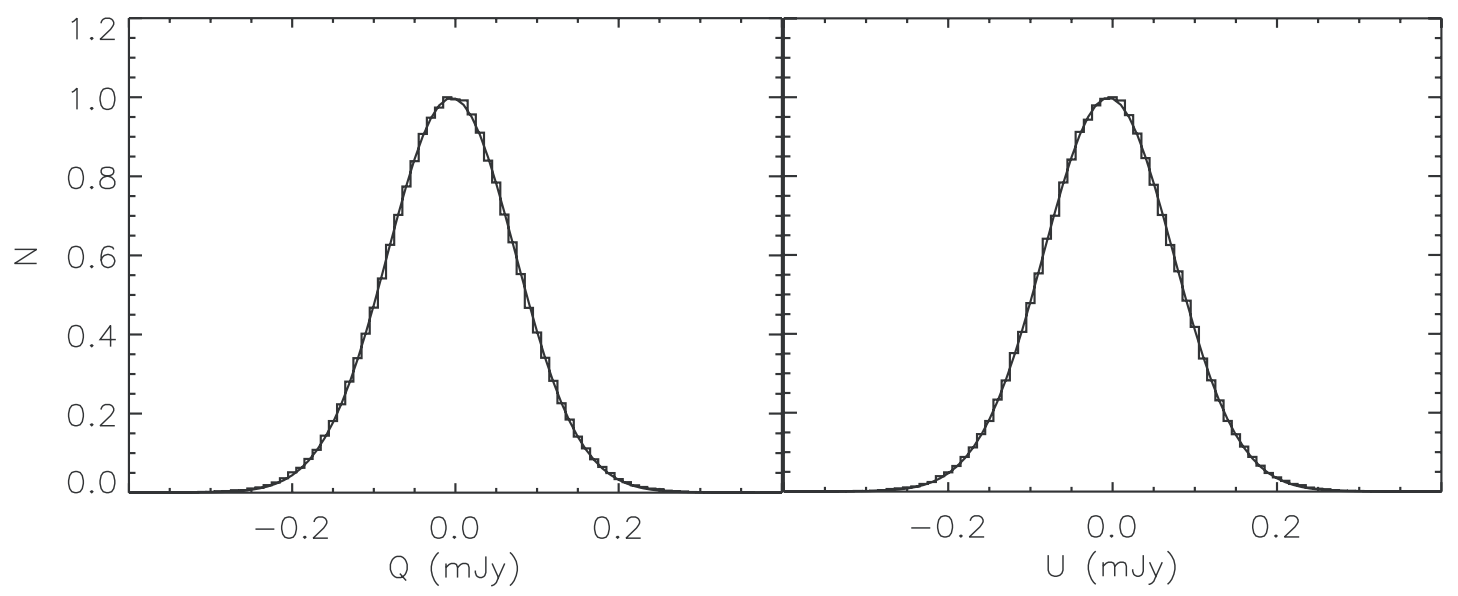

FIG. 3.-Distribution of amplitudes in the $Q$ and $U$ images after dividing by the mosaic weights to produce an image with uniform noise over the map equal to the noise value at the map center. Gaussian fits to the distributions (solid curves) were used to measure the map center rms at $78 \mu \mathrm{Jy}$.

$\alpha=16^{\mathrm{h}} 11^{\mathrm{m}}, \delta=+55^{\circ}(\mathrm{J} 2000.0)$ and cover an area of $7.43 \mathrm{deg}^{2}$ (the area within the thick gray line in Fig. 1). The angular resolution varies with the declination over the images and is given by $b_{\alpha} \times b_{\delta}=49^{\prime \prime} \times 49^{\prime \prime} \operatorname{cosec} \delta$. At the mosaic center the resolution is $49^{\prime \prime} \times 59^{\prime \prime}$. The noise near the center of the mosaic (the white square in Fig. 2) is measured to be $78 \mu \mathrm{Jy}$ in $Q$ and $U$ (see $\S 3.1$ ). The Stokes $I$ image is not limited by confusion, but the noise is slightly higher at $85 \mu \mathrm{Jy}$ near the center of the mosaic, probably because of a contribution from faint sources.

The final images are virtually free of artifacts, so the sensitivity is limited by the noise. The rms brightness sensitivity is $17.4 \mathrm{mK}$. The dynamic range near the center of the mosaic is more than 3000-1 in Stokes I. With very few exceptions, sources in Figure 2 appear as compact (nearly) unresolved sources that can be characterized by their peak intensity and a single polarization angle. This is consistent with expectations from the angular size-flux density relation for extragalactic radio sources (Windhorst 2003); the median angular size of a 1 Jy source is $\sim 10^{\prime \prime}$.

\section{COMPACT POLARIZED SOURCES}

\subsection{Source Detection}

Flux densities and positions of all sources in the pilot deep field images were measured with a source extraction algorithm that fits a two-dimensional Gaussian and a background level to each source. The mosaic images were multiplied by the primary beam response function of the mosaic to obtain an image with a uniform noise level, equal to the noise level at the center of the image. This operation retains the correct signal-to-noise ratio for each source, but the resulting uniform noise level greatly facilitates automated source extraction. The inverse primary beam correction is applied to the measured flux densities of sources from the uniform noise map to transform back to true flux density. The rms noise level in the uniform noise images was measured by fitting a Gaussian function to the distribution of amplitudes in the image. The result is shown in Figure 3. The noise distribution in both the $Q$ and $U$ images is well fitted by a Gaussian with dispersion $\sigma=78 \mu \mathrm{Jy}$.

The polarized flux density image $\left[p=\left(Q^{2}+U^{2}\right)^{1 / 2}\right]$ was searched for polarized sources. For Gaussian noise in the $Q$ and $U$ images with rms amplitude $\sigma$, the statistical distribution of the noise in a measurement of $p$ for a source with an intrinsic polar- ized flux density of $p_{0}$ is a Rice distribution (Rice 1945; Vinokur 1965; Simmons \& Stewart 1985),

$$
f\left(p \mid p_{0}\right)=\frac{p}{\sigma} \exp \left[-\frac{\left(p^{2}+p_{0}^{2}\right)}{2 \sigma^{2}}\right] I_{0}\left(\frac{p p_{0}}{\sigma^{2}}\right) .
$$

Here $I_{0}$ is the modified Bessel function of the first kind. For $p_{0}=0$, the distribution reduces to a Rayleigh distribution,

$$
f(p \mid 0)=\frac{p}{\sigma} \exp \left(-\frac{p^{2}}{2 \sigma^{2}}\right),
$$

which gives the probability distribution of pixel amplitudes in the $p$ image in the absence of polarized emission. The noise in the $p$ image has a nonzero mean and has higher probability of positive peaks above a given detection threshold than Gaussian noise. We searched the $p$ map down to a level of $4.55 \sigma$, which has an equivalent probability for false-positive signals to the $4 \sigma$ level for a Gaussian distribution. The measured polarized flux density $p$ was corrected for noise bias to obtain an estimate of $p_{0}$ through the relation $p_{0}^{2}=p^{2}-\sigma^{2}$, which is a good approximation if the signal-to-noise ratio is larger than 4 (Simmons \& Stewart 1985).

The 83 sources detected are listed in Table 1, which gives the position of each source, the integrated total flux density, noisebias-corrected peak polarized intensity, polarization position angle, fractional polarization defined as the ratio of the bias-corrected peak polarized intensity to the peak total intensity, and the spectral index of the total flux density between 325 and $1420 \mathrm{MHz}$ if the source appears in the WENSS (Westerbork Northern Sky Survey) catalog (Rengelink et al. 1997). To ensure that possible spurious polarized sources due to instrumental polarization are not included, we conservatively remove sources with observed fractional polarization $\Pi$ less than $1 \%$. Only two sources were removed from the sample for this reason.

\subsection{Distribution of Fractional Polarization}

The intrinsic fractional polarization $\Pi_{0}$ of radio sources provides astrophysical information about the nature of the polarized sources. However, the observed fractional polarization $\Pi$ is sensitive to the noise in $p$ and in $I$. In addition to $p$ being a biased estimator of $p_{0}$, the error distribution of the ratio $p / I$ has strong non-Gaussian wings, so $\Pi$ is not a very accurate estimate of $\Pi_{0}$ 
TABLE 1

ELAIS N1 Polarized Sources

\begin{tabular}{|c|c|c|c|c|c|c|c|}
\hline Source Number & $\begin{array}{c}\text { R.A. } \\
\text { (J2000.0) }\end{array}$ & $\begin{array}{c}\text { Decl. } \\
\text { (J2000.0) }\end{array}$ & $\begin{array}{c}I \\
(\mathrm{mJy})\end{array}$ & $\begin{array}{c}p_{0} \\
\text { (mJy beam }^{-1} \text { ) }\end{array}$ & $\begin{array}{l}\text { P.A. } \\
(\text { deg) }\end{array}$ & $\begin{array}{l}\Pi_{0} \\
(\%)\end{array}$ & $\alpha_{325-1420}$ \\
\hline 1 & $160205.52 \pm 0.29$ & $545443.2 \pm 3.0$ & $16.82 \pm 0.56$ & $1.74 \pm 0.11$ & 26 & $10.4 \pm 0.7$ & $-0.78 \pm 0.06$ \\
\hline$\ldots$ & $160234.37 \pm 0.48$ & $545401.8 \pm 4.2$ & $7.95 \pm 0.40$ & $0.98 \pm 0.16$ & 37 & $11.8 \pm 1.9$ & $-0.75 \pm 0.11$ \\
\hline 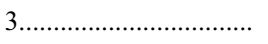 & $160242.00 \pm 0.44$ & $551001.2 \pm 4.4$ & $8.51 \pm 0.78$ & $0.78 \pm 0.10$ & 71 & $19.9 \pm 2.7$ & $-0.23 \pm 0.22$ \\
\hline 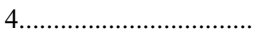 & $160311.52 \pm 0.34$ & $553907.2 \pm 3.8$ & $4.65 \pm 0.40$ & $1.37 \pm 0.11$ & 27 & $28.2 \pm 2.4$ & $>-0.64$ \\
\hline 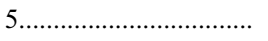 & $160334.08 \pm 0.44$ & $542920.4 \pm 3.9$ & $4.53 \pm 0.37$ & $0.79 \pm 0.10$ & 36 & $17.2 \pm 2.3$ & $-1.46 \pm 0.07$ \\
\hline 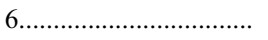 & $160442.24 \pm 0.14$ & $543845.6 \pm 1.4$ & $19.42 \pm 0.55$ & $1.88 \pm 0.11$ & 33 & $10.5 \pm 0.7$ & $-0.99 \pm 0.04$ \\
\hline 7 & $160505.52 \pm 0.15$ & $550046.8 \pm 1.4$ & $27.86 \pm 0.74$ & $1.25 \pm 0.11$ & 7 & $5.3 \pm 0.5$ & $-1.33 \pm 0.03$ \\
\hline 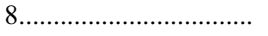 & $160523.04 \pm 0.20$ & $542931.2 \pm 1.8$ & $6.39 \pm 0.29$ & $1.16 \pm 0.11$ & -12 & $17.0 \pm 1.6$ & $>-0.43$ \\
\hline 9 & $160538.88 \pm 0.04$ & $543928.8 \pm 0.4$ & $187.61 \pm 4.74$ & $8.42 \pm 0.24$ & 21 & $5.0 \pm 0.2$ & $-1.00 \pm 0.03$ \\
\hline 10 & $160538.88 \pm 0.20$ & $544127.6 \pm 1.9$ & $2.00 \pm 0.35$ & $1.28 \pm 0.11$ & -37 & $47.3 \pm 4.5$ & $>-1.22$ \\
\hline 11 & $160601.44 \pm 0.04$ & $545410.8 \pm 0.5$ & $209.87 \pm 5.29$ & $5.24 \pm 0.17$ & 15 & $2.9 \pm 0.1$ & $-0.91 \pm 0.03$ \\
\hline 12 & $160608.40 \pm 0.15$ & $551604.8 \pm 1.6$ & $5.06 \pm 0.28$ & $0.86 \pm 0.10$ & 16 & $15.8 \pm 2.0$ & $-0.69 \pm 0.19$ \\
\hline $13 \ldots$ & $160613.44 \pm 0.23$ & $550158.8 \pm 2.3$ & $1.14 \pm 0.18$ & $0.71 \pm 0.11$ & -14 & $52.6 \pm 9.3$ & $>-1.59$ \\
\hline 14 & $160635.52 \pm 0.10$ & $543502.4 \pm 1.0$ & $15.96 \pm 0.51$ & $1.61 \pm 0.11$ & 44 & $12.1 \pm 0.9$ & $-0.73 \pm 0.06$ \\
\hline 15 & $160658.80 \pm 0.32$ & $544312.0 \pm 3.0$ & $4.31 \pm 0.21$ & $0.41 \pm 0.10$ & -9 & $8.6 \pm 2.2$ & $>-0.69$ \\
\hline 16 & $160722.56 \pm 0.22$ & $553104.8 \pm 2.8$ & $12.16 \pm 0.45$ & $0.73 \pm 0.10$ & 1 & $8.0 \pm 1.2$ & $-1.05 \pm 0.05$ \\
\hline 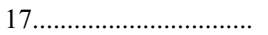 & $160821.36 \pm 0.11$ & $561351.6 \pm 0.8$ & $221.05 \pm 5.59$ & $3.65 \pm 0.15$ & 54 & $2.0 \pm 0.1$ & $0.30 \pm 0.03$ \\
\hline 18 & $160828.56 \pm 0.28$ & $541037.2 \pm 3.7$ & $19.94 \pm 0.56$ & $0.59 \pm 0.10$ & 26 & $3.0 \pm 0.5$ & $-0.68 \pm 0.06$ \\
\hline 19 & $160838.64 \pm 0.29$ & $541434.8 \pm 3.3$ & $2.41 \pm 0.21$ & $0.51 \pm 0.10$ & -19 & $17.0 \pm 3.5$ & $>-1.09$ \\
\hline $20 \ldots \ldots \ldots \ldots \ldots$ & $160847.76 \pm 0.20$ & $561116.8 \pm 1.6$ & $27.09 \pm 1.03$ & $1.66 \pm 0.11$ & 8 & $7.8 \pm 0.6$ & $-1.19 \pm 0.03$ \\
\hline 21 & $160858.32 \pm 0.36$ & $555627.6 \pm 2.2$ & $9.29 \pm 0.48$ & $0.69 \pm 0.10$ & 69 & $12.0 \pm 1.8$ & $-0.82 \pm 0.09$ \\
\hline $22 \ldots \ldots$ & $160904.32 \pm 0.26$ & $561033.6 \pm 2.2$ & $12.68 \pm 0.60$ & $1.33 \pm 0.11$ & 23 & $11.9 \pm 1.0$ & $-1.13 \pm 0.05$ \\
\hline $23 \ldots \ldots$ & $160911.04 \pm 0.18$ & $552631.2 \pm 2.2$ & $4.51 \pm 0.22$ & $0.78 \pm 0.10$ & 4 & $16.9 \pm 2.3$ & $-0.86 \pm 0.17$ \\
\hline 24 & $160922.80 \pm 0.15$ & $56 \quad 1503.6 \pm 1.4$ & $32.46 \pm 0.95$ & $2.14 \pm 0.12$ & 21 & $8.0 \pm 0.5$ & $-0.63 \pm 0.04$ \\
\hline $25, \ldots \ldots \ldots$ & $160931.68 \pm 0.12$ & $552504.8 \pm 1.3$ & $15.32 \pm 0.47$ & $1.41 \pm 0.11$ & 25 & $11.4 \pm 0.9$ & $-1.05 \pm 0.05$ \\
\hline 26 & $160936.24 \pm 0.18$ & $552703.6 \pm 1.5$ & $10.95 \pm 0.37$ & $1.12 \pm 0.10$ & 8 & $11.7 \pm 1.1$ & $-0.71 \pm 0.09$ \\
\hline 27 & $160944.40 \pm 0.38$ & $543751.6 \pm 2.9$ & $6.61 \pm 0.24$ & $0.35 \pm 0.10$ & 59 & $5.3 \pm 1.6$ & $>-0.40$ \\
\hline 28 & $160952.56 \pm 0.24$ & $550708.4 \pm 2.5$ & $0.68 \pm 0.14$ & $0.42 \pm 0.10$ & 1 & $44.1 \pm 12.0$ & $>-1.95$ \\
\hline $29 . \ldots \ldots \ldots$ & $161003.12 \pm 0.10$ & $555237.2 \pm 1.1$ & $96.83 \pm 2.47$ & $1.59 \pm 0.11$ & 30 & $2.0 \pm 0.2$ & $-0.79 \pm 0.03$ \\
\hline $30 \ldots \ldots \ldots$ & $161027.12 \pm 0.14$ & $541254.0 \pm 1.6$ & $8.51 \pm 0.28$ & $1.24 \pm 0.11$ & 30 & $14.4 \pm 1.3$ & $-0.43 \pm 0.17$ \\
\hline $31 \ldots \ldots$ & $161057.84 \pm 0.08$ & $553524.0 \pm 0.7$ & $19.46 \pm 0.53$ & $1.61 \pm 0.11$ & 5 & $9.7 \pm 0.7$ & $-0.63 \pm 0.06$ \\
\hline 32 & $161100.48 \pm 0.06$ & $544203.6 \pm 0.6$ & $29.41 \pm 0.76$ & $2.03 \pm 0.11$ & 19 & $7.8 \pm 0.5$ & $-0.74 \pm 0.04$ \\
\hline 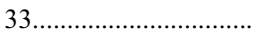 & $161120.40 \pm 0.08$ & $552844.4 \pm 0.9$ & $18.22 \pm 0.50$ & $1.39 \pm 0.11$ & 3 & $8.8 \pm 0.7$ & $-0.88 \pm 0.05$ \\
\hline 34 & $161121.12 \pm 0.30$ & $543155.2 \pm 3.3$ & $3.62 \pm 0.34$ & $0.43 \pm 0.10$ & -5 & $9.9 \pm 2.4$ & $>-0.81$ \\
\hline $35 \ldots \ldots$ & $161129.04 \pm 0.29$ & $555136.0 \pm 2.6$ & $13.22 \pm 0.41$ & $0.44 \pm 0.10$ & 25 & $4.3 \pm 1.0$ & $-0.87 \pm 0.06$ \\
\hline 36 & $161137.92 \pm 0.29$ & $535934.8 \pm 4.2$ & $13.82 \pm 0.44$ & $0.82 \pm 0.10$ & -18 & $5.9 \pm 0.8$ & $-1.03 \pm 0.05$ \\
\hline 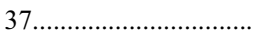 & $161138.16 \pm 0.26$ & $555952.8 \pm 2.7$ & $4.50 \pm 0.23$ & $0.48 \pm 0.10$ & 14 & $11.3 \pm 2.4$ & $-1.19 \pm 0.11$ \\
\hline 38............................ & $161150.88 \pm 0.22$ & $550054.0 \pm 1.0$ & $9.14 \pm 0.41$ & $0.82 \pm 0.10$ & 14 & $16.3 \pm 2.1$ & $-0.63 \pm 0.12$ \\
\hline 39.......................... & $161212.24 \pm 0.02$ & $552248.0 \pm 0.2$ & $312.36 \pm 7.86$ & $20.37 \pm 0.52$ & 29 & $8.0 \pm 0.3$ & $-1.11 \pm 0.03$ \\
\hline 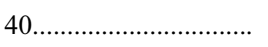 & $161224.00 \pm 0.13$ & $552602.4 \pm 1.5$ & $9.57 \pm 0.34$ & $0.74 \pm 0.10$ & 32 & $8.7 \pm 1.2$ & $-0.56 \pm 0.12$ \\
\hline $41 \ldots$ & $161228.56 \pm 0.34$ & $550646.8 \pm 2.0$ & $10.06 \pm 0.30$ & $0.37 \pm 0.10$ & 59 & $4.1 \pm 1.1$ & $-1.13 \pm 0.06$ \\
\hline $42 \ldots \ldots$ & $161231.68 \pm 0.31$ & $541810.8 \pm 2.5$ & $9.81 \pm 0.36$ & $0.43 \pm 0.10$ & 21 & $4.5 \pm 1.1$ & $-0.73 \pm 0.10$ \\
\hline 43............................... & $161235.28 \pm 0.04$ & $562819.2 \pm 0.4$ & $176.88 \pm 4.51$ & $11.47 \pm 0.31$ & 39 & $7.8 \pm 0.3$ & $-0.96 \pm 0.03$ \\
\hline 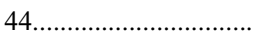 & $161247.52 \pm 0.33$ & $550231.2 \pm 2.9$ & $7.68 \pm 0.25$ & $0.35 \pm 0.10$ & 51 & $4.9 \pm 1.4$ & $-0.74 \pm 0.12$ \\
\hline $45 \ldots \ldots \ldots \ldots \ldots \ldots \ldots \ldots$ & $161251.36 \pm 0.20$ & $560350.4 \pm 2.4$ & $2.59 \pm 0.19$ & $0.61 \pm 0.10$ & 25 & $20.9 \pm 3.7$ & $>-1.04$ \\
\hline 46 & $161302.40 \pm 0.15$ & $543227.6 \pm 1.3$ & $6.48 \pm 0.25$ & $0.73 \pm 0.10$ & 56 & $11.5 \pm 1.7$ & $-0.86 \pm 0.12$ \\
\hline 47 & $161316.80 \pm 0.36$ & $\begin{array}{lll}56 & 08 & 13.2 \pm 3.8\end{array}$ & $3.85 \pm 0.36$ & $0.56 \pm 0.10$ & -28 & $14.1 \pm 2.6$ & $>-0.77$ \\
\hline 48 & $161319.20 \pm 0.09$ & $541640.8 \pm 1.0$ & $5.78 \pm 0.43$ & $1.34 \pm 0.11$ & 5 & $31.5 \pm 2.8$ & $>-0.50$ \\
\hline 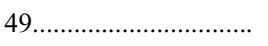 & $161325.92 \pm 0.24$ & $553939.6 \pm 2.4$ & $3.06 \pm 0.18$ & $0.35 \pm 0.10$ & 17 & $12.2 \pm 3.6$ & $>-0.93$ \\
\hline 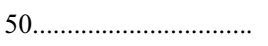 & $161326.64 \pm 0.10$ & $551546.8 \pm 1.1$ & $14.60 \pm 0.14$ & $0.80 \pm 0.10$ & 14 & $6.5 \pm 0.9$ & $-1.19 \pm 0.04$ \\
\hline $51 \ldots \ldots \ldots$ & $161328.80 \pm 0.27$ & $561749.2 \pm 2.1$ & $17.29 \pm 0.57$ & $0.87 \pm 0.10$ & 26 & $6.8 \pm 0.8$ & $-0.53 \pm 0.07$ \\
\hline $52 \ldots \ldots \ldots \ldots \ldots \ldots \ldots$ & $161330.72 \pm 0.06$ & $542721.6 \pm 0.6$ & $80.29 \pm 2.03$ & $2.67 \pm 0.12$ & 15 & $3.7 \pm 0.2$ & $-0.85 \pm 0.03$ \\
\hline 53 & $161336.72 \pm 0.36$ & $541116.8 \pm 2.7$ & $1.70 \pm 0.36$ & $0.59 \pm 0.10$ & 1 & $22.9 \pm 4.2$ & $>-1.33$ \\
\hline 54 & $161341.76 \pm 0.19$ & $561149.2 \pm 1.4$ & $101.05 \pm 7.62$ & $2.80 \pm 0.12$ & 22 & $10.9 \pm 0.6$ & $0.09 \pm 0.04$ \\
\hline 55 & $161348.48 \pm 0.27$ & $5414 \quad 13.2 \pm 2.5$ & $8.12 \pm 0.40$ & $0.64 \pm 0.10$ & 18 & $7.3 \pm 1.2$ & $-1.10 \pm 0.07$ \\
\hline 56 & $161356.16 \pm 0.25$ & $545728.8 \pm 2.5$ & $0.45 \pm 0.09$ & $0.28 \pm 0.10$ & -1 & $50.6 \pm 19.2$ & $-3.97 \pm 0.03$ \\
\hline 57 & $161356.84 \pm 0.22$ & $550208.2 \pm 2.3$ & $0.81 \pm 0.13$ & $0.50 \pm 0.02$ & -31 & $49.8 \pm 5.7$ & $>-1.82$ \\
\hline 58 & $161400.96 \pm 0.20$ & $535721.6 \pm 1.8$ & $14.75 \pm 0.49$ & $1.08 \pm 0.10$ & 32 & $7.5 \pm 0.8$ & $-0.41 \pm 0.10$ \\
\hline 59 & $161416.80 \pm 0.29$ & $554257.6 \pm 3.5$ & $1.14 \pm 0.36$ & $0.46 \pm 0.10$ & 56 & $64.0 \pm 17.2$ & $>-1.60$ \\
\hline $60 \ldots \ldots \ldots \ldots \ldots$ & $161421.12 \pm 0.06$ & $553639.6 \pm 0.6$ & $35.41 \pm 0.93$ & $3.00 \pm 0.13$ & 18 & $9.9 \pm 0.5$ & $-0.74 \pm 0.04$ \\
\hline 61 & $161432.64 \pm 0.20$ & $553831.2 \pm 2.5$ & $1.27 \pm 0.39$ & $0.47 \pm 0.10$ & -79 & $90.8 \pm 27.5$ & $>-1.52$ \\
\hline $62 \ldots \ldots \ldots \ldots \ldots \ldots \ldots$ & $161527.36 \pm 0.13$ & $542710.8 \pm 1.2$ & $4.51 \pm 0.20$ & $0.97 \pm 0.10$ & 5 & $20.1 \pm 2.3$ & $>-0.66$ \\
\hline $63 \ldots \ldots$ & $161530.96 \pm 0.16$ & $545230.0 \pm 2.0$ & $5.43 \pm 0.22$ & $0.68 \pm 0.10$ & 20 & $13.1 \pm 2.0$ & $-1.16 \pm 0.09$ \\
\hline 64................................. & $161536.72 \pm 0.19$ & $534637.2 \pm 2.4$ & $54.20 \pm 1.46$ & $2.64 \pm 0.12$ & 19 & $5.6 \pm 0.3$ & $-0.85 \pm 0.03$ \\
\hline 65 & $161549.68 \pm 0.06$ & $551640.8 \pm 0.7$ & $27.80 \pm 0.72$ & $1.75 \pm 0.11$ & 35 & $7.4 \pm 0.5$ & $-0.68 \pm 0.04$ \\
\hline
\end{tabular}


TABLE 1-Continued

\begin{tabular}{|c|c|c|c|c|c|c|c|}
\hline Source Number & $\begin{array}{c}\text { R.A. } \\
(\text { J2000.0) }\end{array}$ & $\begin{array}{c}\text { Decl. } \\
(\mathrm{J} 2000.0)\end{array}$ & $\begin{array}{c}I \\
(\mathrm{mJy})\end{array}$ & $\begin{array}{c}p_{0} \\
\left.\text { (mJy beam }^{-1}\right)\end{array}$ & $\begin{array}{l}\text { P.A. } \\
\text { (deg) }\end{array}$ & $\begin{array}{l}\Pi_{0} \\
(\%)\end{array}$ & $\alpha_{325-1420}$ \\
\hline 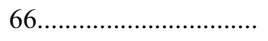 & $161623.04 \pm 0.14$ & $552700.0 \pm 1.3$ & $13.10 \pm 0.40$ & $0.90 \pm 0.10$ & 27 & $8.8 \pm 1.0$ & $-0.89 \pm 0.06$ \\
\hline 67....................... & $161623.52 \pm 0.17$ & $545743.2 \pm 1.6$ & $10.35 \pm 0.48$ & $0.60 \pm 0.10$ & 10 & $6.0 \pm 1.0$ & $-0.97 \pm 0.07$ \\
\hline 68 & $161637.92 \pm 0.18$ & $\begin{array}{ll}55 & 45 \quad 14.4 \pm 2.1\end{array}$ & $74.66 \pm 1.90$ & $0.85 \pm 0.10$ & 51 & $1.3 \pm 0.2$ & $-0.61 \pm 0.03$ \\
\hline 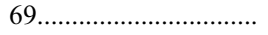 & $161639.36 \pm 0.11$ & 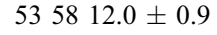 & $351.23 \pm 9.13$ & $5.14 \pm 0.19$ & 23 & $1.7 \pm 0.1$ & $-0.85 \pm 0.03$ \\
\hline 70..................... & $161640.08 \pm 0.18$ & $562038.4 \pm 1.5$ & $18.64 \pm 0.70$ & $1.37 \pm 0.11$ & 24 & $9.3 \pm 0.8$ & $-0.52 \pm 0.07$ \\
\hline 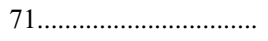 & $161757.60 \pm 0.22$ & $545136.0 \pm 3.0$ & $14.65 \pm 0.46$ & $0.68 \pm 0.10$ & -17 & $5.9 \pm 0.9$ & $-1.11 \pm 0.04$ \\
\hline 72 & $161806.72 \pm 0.36$ & $544246.8 \pm 2.4$ & $5.31 \pm 0.26$ & $0.65 \pm 0.10$ & 24 & $12.3 \pm 2.0$ & $>-0.55$ \\
\hline 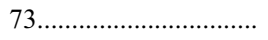 & $161832.64 \pm 0.05$ & $543144.4 \pm 0.5$ & $48.68 \pm 1.27$ & $4.56 \pm 0.15$ & -1 & $10.3 \pm 0.4$ & $-0.78 \pm 0.03$ \\
\hline 74 & $161857.57 \pm 0.12$ & $542926.2 \pm 1.3$ & $132.06 \pm 5.88$ & $2.55 \pm 0.14$ & 21 & $2.3 \pm 0.2$ & $-1.39 \pm 0.03$ \\
\hline 75 & $161859.28 \pm 0.18$ & $545240.8 \pm 1.7$ & $40.08 \pm 1.07$ & $1.86 \pm 0.11$ & 21 & $6.0 \pm 0.4$ & $-0.50 \pm 0.04$ \\
\hline $76 \ldots \ldots \ldots \ldots$ & $161915.36 \pm 0.20$ & $\begin{array}{lll}55 & 05 & 13.2 \pm 1.4\end{array}$ & $16.65 \pm 0.50$ & $0.69 \pm 0.10$ & -2 & $5.7 \pm 0.9$ & $-0.30 \pm 0.11$ \\
\hline 77. & $161919.20 \pm 0.08$ & $553556.4 \pm 0.8$ & $53.48 \pm 1.36$ & $1.98 \pm 0.11$ & 15 & $4.1 \pm 0.3$ & $-0.44 \pm 0.04$ \\
\hline 78. & $161919.44 \pm 0.06$ & $544825.2 \pm 0.6$ & $57.59 \pm 1.46$ & $3.32 \pm 0.13$ & 11 & $6.1 \pm 0.3$ & $-0.67 \pm 0.03$ \\
\hline 79 & $161924.24 \pm 0.18$ & $555052.8 \pm 1.6$ & $2.18 \pm 0.21$ & $0.79 \pm 0.10$ & 19 & $26.8 \pm 3.7$ & $>-1.16$ \\
\hline $80 \ldots \ldots \ldots$ & $162113.68 \pm 0.10$ & $552342.0 \pm 0.9$ & $58.11 \pm 1.49$ & $2.62 \pm 0.12$ & 50 & $4.8 \pm 0.3$ & $-0.94 \pm 0.03$ \\
\hline $81 \ldots \ldots \ldots$ & $162118.72 \pm 0.28$ & $553827.6 \pm 2.3$ & $7.97 \pm 0.40$ & $0.74 \pm 0.10$ & 7 & $8.5 \pm 1.2$ & $-0.98 \pm 0.08$ \\
\hline $82 \ldots \ldots \ldots \ldots$ & $162145.36 \pm 0.10$ & $554937.2 \pm 1.0$ & $31.10 \pm 1.01$ & $2.05 \pm 0.11$ & 60 & $6.9 \pm 0.4$ & $-0.62 \pm 0.04$ \\
\hline 83 & $162208.64 \pm 0.14$ & $552428.8 \pm 1.6$ & $14.23 \pm 2.15$ & $4.55 \pm 0.15$ & 6 & $27.7 \pm 1.2$ & $-1.57 \pm 0.03$ \\
\hline
\end{tabular}

even for relatively high signal-to-noise sources. This is illustrated in Figure 4, which shows the relation between $\Pi$ and Stokes $I$ flux density for an artificial sample of sources, all with $\Pi_{0}=5 \%$ and Gaussian noise added with equal amplitudes in Stokes $I, Q$, and $U$. Only those sources with a $p$ flux density more than $5 \sigma$ are shown. Error bars represent standard error propagation in $\Pi$ from the errors in $p$ and $I$. The high values of $\Pi$ at signal-to-noise ratio less than $\sim 100$ is a result of the detection threshold in $p$ and the nonGaussian error statistics of $\Pi$. The effect is much larger than the polarization noise bias alone.

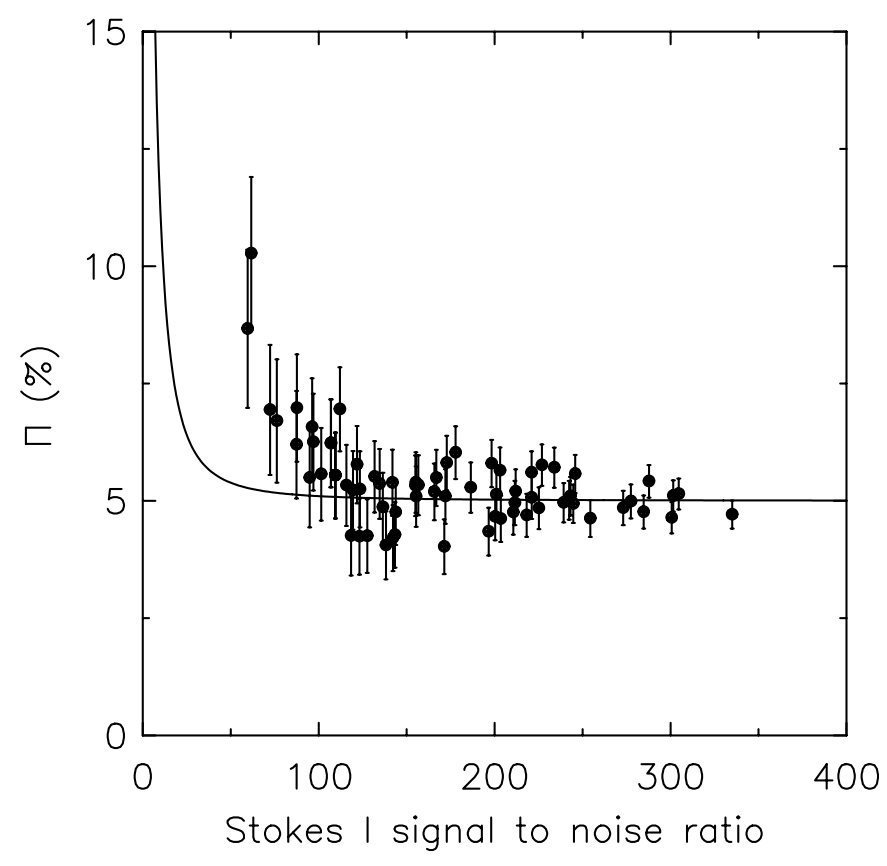

Fig. 4.- Illustration of the effect of noise and the polarized flux density detection threshold on $\Pi$ as a function of flux density. This figure shows the variation of $\Pi$ with flux density for a simulated sample of sources, all $5 \%$ polarized, with random Gaussian noise added in $I, Q$, and $U$. The error bars are derived from standard error propagation, assuming the noise is known. The curve shows the effect of polarization bias on $\Pi$, defined as $\left(p_{0}^{2}+\sigma^{2}\right)^{1 / 2} / I$ (Simmons \& Stewart 1985).
These problems highlight the need for careful analysis of the effects of noise and polarization detection thresholds in studies of the fractional polarization of faint sources. Previous studies have focused on the fractional polarization of bright radio sources in the NVSS. Mesa et al. (2002), Tucci et al. (2004), and Beck \& Gaensler (2004) considered polarized sources in the NVSS with Stokes I flux density stronger than 80,100 , and $80 \mathrm{mJy}$, respectively. The high flux density thresholds in these studies were adopted to achieve a good level of completeness in $\Pi$ down to the limit set by residual instrumental polarization $(\Pi \approx 1 \%)$. These studies found a $\Pi$ distribution that decreases monotonically with increasing $\Pi$, and with a median $\Pi \approx 1.8 \%$. For these bright sources, noise effects are small, and the $\Pi$ distribution should be close to the intrinsic $\Pi_{0}$ distribution down to limits set by residual instrumental polarization.

In this paper we investigate the shape of the $\Pi_{0}$ distribution for much fainter Stokes $I$ flux densities than those considered in previous work. The present data have angular resolution similar to the NVSS, so differences in the measured degree of polarization because of a difference in resolution are not expected. Our results can be compared directly with results based on the NVSS.

Noise effects such as those illustrated in Figure 4 were taken into account by a Monte Carlo analysis. A set of simulated catalogs was generated to accurately represent the effects of noise and the polarized flux density detection threshold in the data. Stokes I flux densities of simulated sources were drawn from the fit to observed source counts by Windhorst et al. (1990). We assume in these simulations that the Stokes $I$ source counts of polarized sources have the same shape as those for all radio sources. This is a reasonable assumption because $\sim 80 \%$ of radio sources in the NVSS display significant polarization (Mesa et al. 2002; Tucci et al. 2004; Beck \& Gaensler 2004). The intrinsic Stokes $I$ flux density is multiplied by the degree of polarization $\Pi_{0}$ drawn from an assumed $\Pi_{0}$ distribution to obtain the intrinsic polarized intensity $p_{0}$.

The intrinsic Stokes $I_{0}$ and $p_{0}$ of a simulated source are transformed to observed flux densities $I$ and $p$ by adding noise with statistical properties identical to the properties of the noise in the data. First, $p_{0}$ is converted into intrinsic Stokes $Q_{0}$ and $U_{0}$, assuming a random polarization angle. The error in the flux density is assumed to consist of a part that is proportional to the noise in 


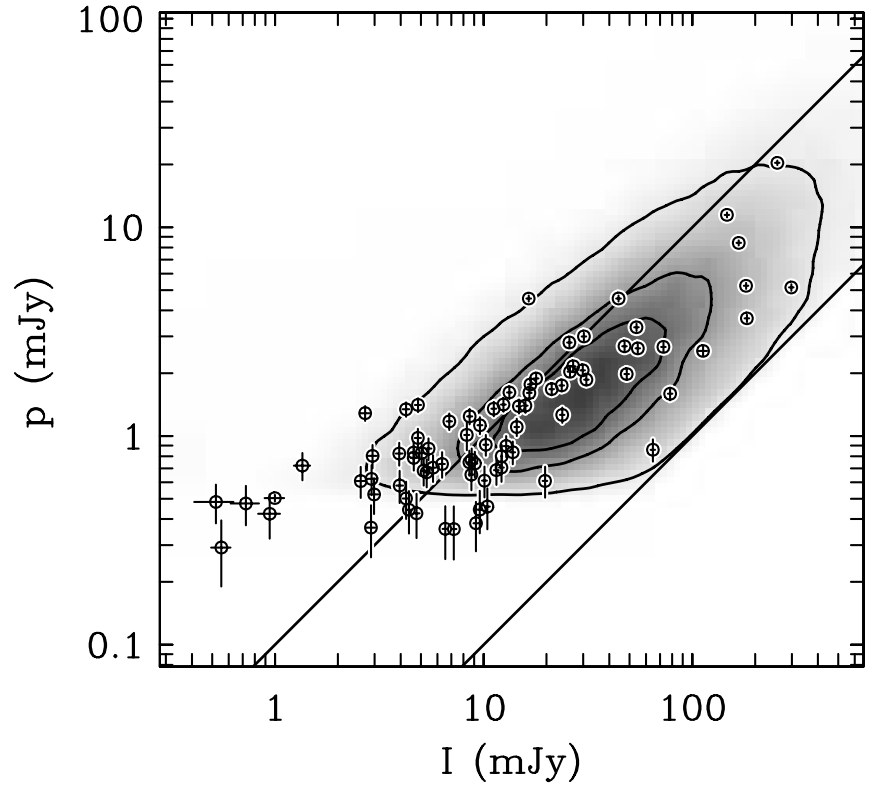

FIG. 5.-Distribution in $\log I-\log p$ of observed sources (open circles), compared with the simulated distribution that assumes the $\Pi_{0}$ distribution of Beck \& Gaensler (2004). Gray scales and contours show the two-dimensional probability density function of sources in the simulated catalog. The inner contours enclose $25 \%$ and $50 \%$ of the simulated sources, while the outer contour encloses $90 \%$. Two lines mark the loci of sources with $\Pi=1 \%$ (right) and $\Pi=10 \%$ (left).

the image at the location of the source, and a part that is proportional to the flux density of the source, added in quadrature. The error in the flux density $S$ is evaluated as

$$
\sigma_{S}=S \sqrt{C_{1}^{2}+C_{2}^{2}\left(\frac{\sigma^{2}}{S^{2}}\right)}
$$

where $S$ represents the intrinsic flux density $I_{0}, Q_{0}$, or $U_{0}, \sigma$ is the rms noise in the image, and $C_{1}$ and $C_{2}$ are constants. The value of $C_{1}$ was determined from the rms variation of the flux density of bright sources in the 10 fields after field registration (Taylor et al. 2003). Flux densities of sources brighter than $100 \mathrm{mJy}$ varied by $2.5 \%$ (rms) over the 10 fields. From this we adopt $C_{1}=0.025$. The value of the constant $C_{2}=1.3$ was taken to be that found by Rengelink et al. (1997) from Monte Carlo simulations for flux density errors in the WENSS. The value of $\sigma$ is different for each source, to represent variation of the noise with location in the mosaic. The distribution of polarized intensities of the resulting simulated sources have the same statistical effects as the observed polarized intensities, including the effects of noise bias, the detection threshold, and the variation of the noise with position in the mosaic.

To derive the $\Pi_{0}$ distribution directly from the data, we compare the distribution of the data in a $\log I-\log p$ diagram with the probability distribution for simulated source catalogs. Figure 5 shows the observed data points, and the model probability distribution assuming the $\Pi_{0}$ distribution function of Beck \& Gaensler (2004). As expected, sources brighter than $\sim 80 \mathrm{mJy}$ are represented well by this model. However, fainter sources in our sample are more highly polarized than predicted by this distribution. This is clearly visible in Figure 5 for sources with $10 \mathrm{mJy}<$ $I<30 \mathrm{mJy}$, where an offset exists between the distribution of observed sources and the predicted ridge of maximum source density. A two-dimensional Kolmogorov-Smirnov test (Peacock 1983) rejected the hypothesis that the data were drawn from the simulated distribution at the $99.9 \%$ confidence level.
The best-fit $\Pi_{0}$ distribution was derived by fitting source probability density distributions to the data in the $\log I-\log p$ plane for trial $\Pi_{0}$ distributions. The trial $\Pi_{0}$ distributions were represented by a low-order Gauss-Hermite series, also called a Gram-Charlier series, following the description of van der Marel \& Franx (1993):

$$
f\left(\Pi_{0}\right)=\exp \left(-\frac{\Pi_{0}^{2}}{2 \sigma_{\Pi_{0}}^{2}}\right)\left[1+\sum_{i=3}^{N} h_{i} H_{i}\left(\Pi_{0} / \sigma_{\Pi_{0}}\right)\right] .
$$

We consider only modest deviations from a Gaussian, since previous work on bright NVSS sources suggests that the shape of the distribution is nearly Gaussian. Assuming that the $\Pi_{0}$ distribution peaks at zero and declines monotonically with increasing $\Pi_{0}$, we use only the fourth term $(i=4)$ in equation (4), which results in symmetric deviations. A coefficient $h_{4}>0$ means that the wings of the distribution are stronger than the wings of a Gaussian distribution, as shown graphically by van der Marel \& Franx (1993). Higher order terms were not considered because of the limited size of our data sample at this time. The $\Pi_{0}$ distribution defined by equation (4) thus has two free parameters, the Gaussian standard deviation, $\sigma_{\Pi_{0}}$, and the amplitude of the lowest order symmetric deviation from a Gaussian, $h_{4}$. The fits maximize the likelihood $L$ of the data as a function of these parameters,

$$
L=\prod_{i=1}^{N_{\text {data }}} P\left(I_{i}, p_{i} \mid \sigma_{\Pi_{0}}, h_{4}\right)
$$

with the probability of an observed $\left(I_{i}, p_{i}\right)$ for a given $\sigma_{\Pi_{0}}$ and $h_{4}$,

$$
P\left(I_{i}, p_{i} \mid \sigma_{\Pi_{0}}, h_{4}\right)=\frac{1}{M_{\text {model }}} \sum_{j=1}^{M_{\text {model }}} \exp \left[\frac{\left(I_{i}-I_{j}\right)^{2}}{2 \sigma_{I, i}^{2}}+\frac{\left(p_{i}-p_{j}\right)^{2}}{2 \sigma_{p, i}^{2}}\right] .
$$

The product over $i$ is over all sources in the data, $N_{\text {data }}$, whereas the sum over $j$ is over all simulated sources in the catalog, $M_{\text {model }}$, for a particular $\sigma_{\Pi_{0}}$ and $h_{4}$. Typically $M_{\text {model }} \sim 10^{5}$. The values $\sigma_{I, i}$ and $\sigma_{p, i}$ are the observed errors in Stokes $I$ and polarized flux density for the $i$ th source.

The maximum likelihood $\Pi_{0}$ distribution was found through a grid search over the parameter space. The best-fitting model has parameters $\sigma_{\Pi_{0}}=7 \%$ and $h_{4}=0.05$. The probability density function of this model is shown along with the data in Figure 6. The uncertainty in the best-fitting parameters was evaluated empirically. Three hundred randomly selected samples, each containing on average the same number of sources as the observed sample, were drawn from the best-fitting simulated catalog. Each of these samples was fitted with the maximum likelihood fit to evaluate the spread of the best-fitting parameters. Two-thirds of these fits yielded a $\sigma_{\Pi_{0}}$ within $1 \%$ of the maximum likelihood value $7 \%$. We conclude that $\sigma_{\Pi_{0}}=7.0 \% \pm$ $1.0 \%$. The fitted value of $h_{4}$ is not independent of $\sigma_{\Pi_{0}}$ because a larger $h_{4}$ can partially compensate for a smaller $\sigma_{\Pi_{0}}$. From the same 300 experimental fits, two-thirds yielded a value $h_{4}<0.1$. Although the data are consistent with $h_{4}=0$, those fits with $h_{4}$ constrained to be zero yield an average $\sigma_{\Pi_{0}}=8 \% \pm 1 \%$. The data therefore suggest that the $\Pi_{0}$ distribution may be somewhat broader than a simple Gaussian with $\sigma_{\Pi_{0}}=7.0 \%$.

The best-fitting model was also subjected to a two-dimensional Kolmogorov-Smirnov test. The hypothesis that the data were drawn from the best-fit distribution in Figure 6 was rejected at 


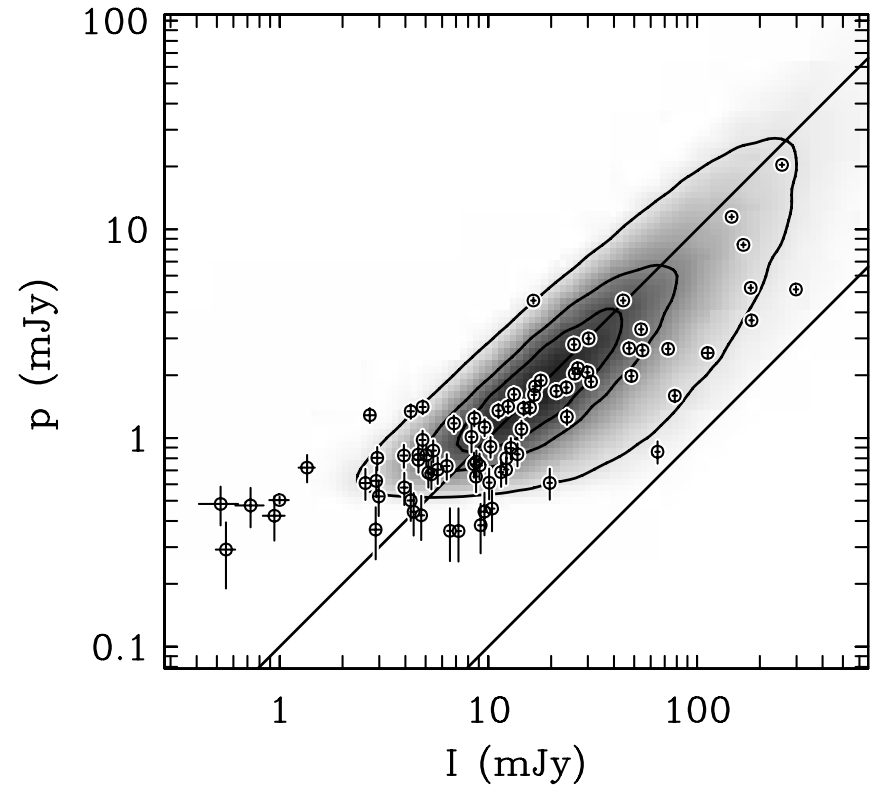

FIG. 6.-Same as Fig. 5, now for the best-fitting Gauss-Hermite $\Pi_{0}$ distribution, with $\sigma_{\Pi_{0}}=7.0 \%$ and $h_{4}=0.05$.

the $98 \%$ confidence level. This is much better than the result for the Beck \& Gaensler (2004) distribution, but it is still suggestive that all the data are not well represented by the maximum likelihood model distribution. This is entirely the result of the fact that the best-fitting Gauss-Hermite distribution does not fit the bright sources in the sample very well. We were able to produce a better fit by creating a set of hybrid simulated catalogs that use the Beck \& Gaensler (2004) distribution for brighter sources and our bestfitting distribution for faint sources. The transition between these regimes was made smooth, with equal contributions from the two distributions at a flux density of $30 \mathrm{mJy}$. These hybrid catalogs fitted the data significantly better, with the best-fitting model, using $\sigma_{\Pi_{0}}=7.0 \%$ and $h_{4}=0.05$ at low flux density, passing the two-dimensional Kolmogorov-Smirnov test. The median fractional polarization of the best-fit $\Pi_{0}$ distribution for polarized sources with Stokes $I$ below $30 \mathrm{mJy}$ is $4.8 \% \pm 0.7 \%$.

\subsection{Source Counts}

To derive source counts of the polarized sources, the effect of the varying noise level over the mosaic on source detection probability (completeness correction) was measured as a function of flux density by repeating the source detection on 1000 simulated images having the same noise characteristics and source density as the data. Separate simulations were performed for total intensity and for polarized intensity images. Each simulated image contained random Gaussian noise smoothed to the resolution of the DRAO images, and the same uniform rms amplitude as the data. For each simulated polarization image, two independent noise images were created to represent the $Q$ and $U$ images. Sources were placed at random positions, with flux densities drawn from the source-count curve derived by Windhorst et al. (1990) between 0.1 and $500 \mathrm{mJy}$. Sources below the detection limit were included in the simulations to account for crowding in the field, and the possibility that faint sources are observed above the detection limit because of noise. The resulting synthetic Stokes $I$ and polarized intensity images were then searched for sources in an identical manner as for the observed images.

Figure 7 shows the derived differential source counts for total intensity $(\log N-\log I)$ and polarized flux density $(\log N-\log p)$

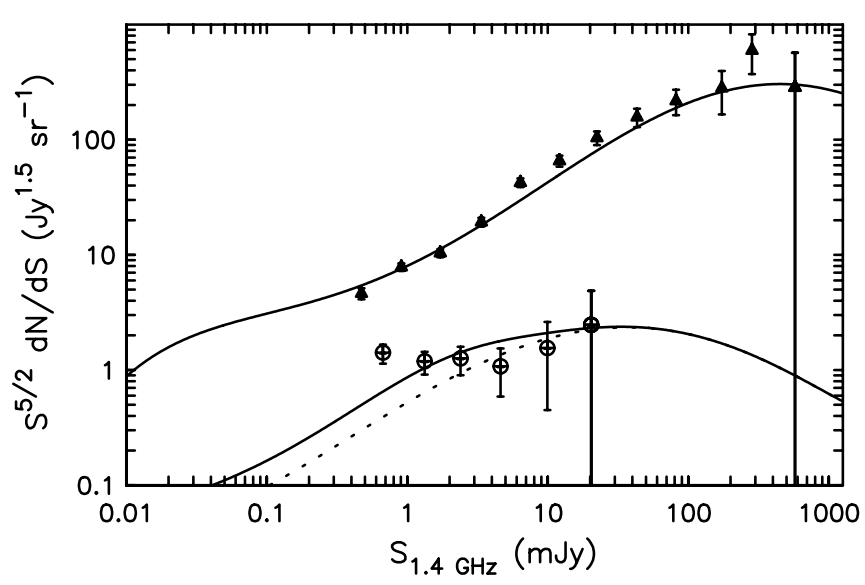

FIG. 7.-Euclidean-normalized source counts for total flux density (triangles) and polarized flux density (circles). The upper solid curve is the fit to observed source counts from Windhorst et al. (1990). The lower solid curve shows polarized source counts predicted by convolving the Stokes $I$ source counts with the $\Pi$ distribution derived from ELAIS N1 data for faint sources and the Beck \& Gaensler (2004) distribution for bright sources, as explained in the text. The dotted curve shows polarized source counts derived by convolving only the Beck \& Gaensler (2004) distribution with the Windhorst et al. (1990) source counts curve.

at $1420 \mathrm{MHz}$, normalized to the Euclidean expectation in the conventional way. The polarized source counts are also listed in Table 2. Counts were derived in bins starting at $500 \mu \mathrm{Jy}$ to avoid sources with completeness correction greater than 10 . This resulted in the removal of the 15 faintest sources from the counts. The Stokes $I$ source counts show good agreement with the Windhorst curve, although we find somewhat higher numbers around $10 \mathrm{mJy}$. The polarized source counts are nearly flat in the flux density range observed, consistent with the increased fractional polarization of the faint radio sources.

Figure 7 shows predicted polarized source count curves derived by convolving Windhorst's Stokes $I$ counts with the strongsource $\Pi_{0}$ distribution from Beck \& Gaensler (2004) (dashed curve), as well as our hybrid $\Pi_{0}$ distribution fit to the $\log I-\log p$ distribution in $\S 3.2$ (solid curve). The observed polarized source counts show an excess over the Beck \& Gaensler (2004) extrapolation for $p<3 \mathrm{mJy}$, and are consistent with the prediction based on the derived higher $\Pi_{0}$ for these sources. The data point at the lowest flux density lies marginally above our predicted curve. This may suggest a continuing trend toward even higher fractional polarization in the submillijansky population. The complete DRAO 30-field survey of the region, and other deep polarization surveys, will test this.

\subsection{Identification with Spitzer Objects}

The positions of the polarized radio sources were examined in the Spitzer SWIRE images of ELAIS N1. Accurate positions for the sources were obtained from the VLA FIRST survey

TABLE 2

Polarized Source Counts

\begin{tabular}{|c|c|c|}
\hline $\begin{array}{c}p \\
(\mathrm{mJy})\end{array}$ & $N$ & $\begin{array}{l}p^{2.5} d N / d p \\
\left(\mathrm{Jy}^{1.5} \mathrm{sr}^{-1}\right)\end{array}$ \\
\hline 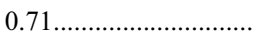 & 29 & $1.38 \pm 0.26$ \\
\hline 1.42 & 20 & $1.26 \pm 0.28$ \\
\hline $2.50 \ldots$ & 11 & $1.10 \pm 0.33$ \\
\hline $4.61 \ldots$ & 5 & $1.01 \pm 0.45$ \\
\hline $9.94 \ldots$ & 2 & $1.46 \pm 1.03$ \\
\hline $20.4 \ldots \ldots \ldots \ldots-\ldots$ & 1 & $2.32 \pm 2.32$ \\
\hline
\end{tabular}




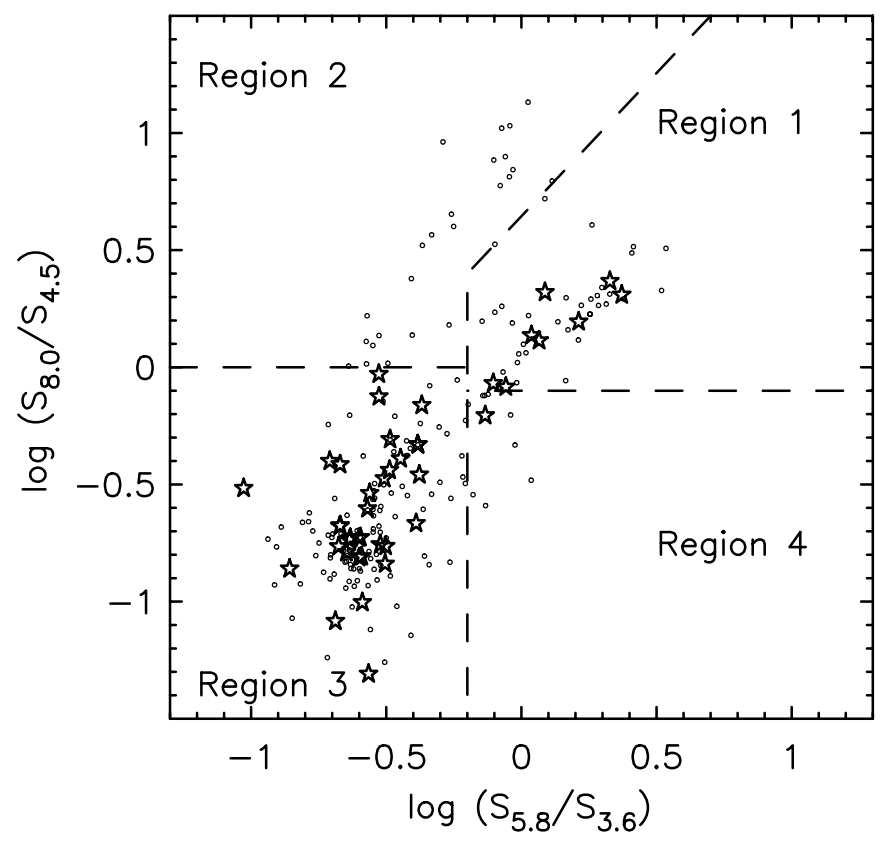

FIG. 8.-Spitzer near-infrared color-color diagram of ELAIS N1 radio source host galaxies. Stars: Polarized sources. Circles: Sources with no detectable polarized emission. The division of this diagram into four regions and their interpretation follows Sajina et al. (2005) and is explained in the text.

images (White et al. 1997), which provides $1.4 \mathrm{GHz}$ continuum Stokes $I$ images at $5^{\prime \prime}$ resolution with a $1 \sigma$ sensitivity of $150 \mu \mathrm{Jy}$ beam $^{-1}$. The sensitivity of the FIRST images is sufficient to detect a Stokes $I$ counterpart for every polarized source in the ELAIS N1 deep field. Approximately $35 \%$ of the polarized radio sources showed resolved structure on scales of $2^{\prime \prime}-30^{\prime \prime}$ in the FIRST images. A polarized source may be associated with a radio lobe instead of the core of a radio galaxy. Visual inspection avoided misidentification in such cases. As a comparison to the polarized sources we also searched the SWIRE images for identification of sources with Stokes $I$ flux density larger than $1 \mathrm{mJy}$ but no detectable polarization.

In total, 54 polarized sources were unambiguously identified with Spitzer objects. Another 17 polarized sources had uncertain identifications; i.e., there was more than one possible counterpart within the errors of the radio position. Two sources were not covered by SWIRE. The remaining $12 \%$ of the sources ( 10 objects) have no counterpart in the SWIRE images. Similar statistics resulted from the search for counterparts of the Stokes $I$ sources with no detectable polarized emission. A wide range in flux density and angular size was found among the identified Spitzer galaxies. Although some faint galaxies appear unresolved in the Spitzer images, the identified polarized sources seem to be mainly associated with elliptical galaxies. Three of the polarized sources have counterparts in the Spitzer images that are too faint to appear in the Spitzer ELAIS N1 source catalog. These sources were not included in the subsequent analysis.

Figure 8 shows a near-infrared color-color diagram of Spitzer galaxies identified with radio sources that had cataloged flux densities in all four IRAC bands at 3.6, 4.5, 5.8, and $8.0 \mu \mathrm{m}$. This includes 41 of the polarized sources. Since many of the sources are identified with extended galaxies, we used the isophotal fluxes in each band. Our analysis of this color-color diagram is based on the modeling of Sajina et al. (2005), which divided the diagram into the four regions separated by dashed lines in Figure 8. Boundaries between these regions were defined so as to separate galaxies
TABLE 3

Radio Sources in the IRAC Color-Color Diagram

\begin{tabular}{|c|c|c|c|c|}
\hline \multirow[b]{2}{*}{ REGION } & \multicolumn{2}{|c|}{ Polarization Detected } & \multicolumn{2}{|c|}{ OTHER SOURCES } \\
\hline & Number & Percent & Number & Percent \\
\hline $1 \ldots \ldots \ldots \ldots \ldots \ldots \ldots \ldots \ldots$ & 8 & $20 \pm 7$ & 32 & $19 \pm 3$ \\
\hline $2 \ldots \ldots \ldots \ldots \ldots \ldots \ldots \ldots \ldots \ldots \ldots \ldots \ldots \ldots \ldots \ldots$ & 0 & 0 & 25 & $15 \pm 3$ \\
\hline $3 a^{a} \ldots$ & 21 & $51 \pm 11$ & 80 & $47 \pm 5$ \\
\hline ............ & 11 & $27 \pm 8$ & 24 & $14 \pm 3$ \\
\hline ................. & 1 & $2 \pm 2$ & 9 & $5 \pm 2$ \\
\hline
\end{tabular}

a Region 3 blue clump, selected by $\log \left(S_{8.0} / S_{4.5}\right) \leqq-0.5$.

b Region 3 PAH, selected by $\log \left(S_{8.0} / S_{4.5}\right)>-0.5$.

depending on the strength of near-infrared PAH bands and the slope of the near-infrared continuum. Region 1 is mainly populated by sources with a continuum that rises with wavelength. The near-infrared spectrum of these sources is usually dominated by nonequilibrium emission of stochastically heated very small dust grains, interpreted as PAH destruction by the hard ultraviolet spectrum of an AGN. Region 2 is occupied mainly by dusty starforming galaxies with strong PAH bands at redshift $z<0.5$. This is because the strongest PAH features at low redshift contribute to the flux in the IRAC bands at 3.6 and $8.0 \mu \mathrm{m}$. Region 3 is occupied by galaxies with fainter PAH emission or by dusty star-forming galaxies at redshift $z=0.5-1.5$. A conspicuous concentration of galaxies in region 3 is the blue clump near $\log \left(S_{5.8} / S_{3.6}\right)=-0.6, \log \left(S_{8.0} / S_{4.5}\right)=-0.8$. Galaxies in the blue clump have a near-infrared continuum that declines with wavelength, since the spectrum is dominated by the starlight of an old stellar population. These are elliptical galaxies at a wide range of redshift. Region 4 is populated by PAH-dominated sources at redshift $z=1.5-2$.

The number of sources by region in Figure 8 is listed in Table 3. The host galaxies of the polarized sources occupy mainly regions 1 and 3 . The source in region 4 cannot be considered a convincing high-redshift galaxy in view of uncertainties in the photometry and its proximity to the boundary with regions 1 and 3 . The majority (71\%) of the host galaxies of polarized sources in the Spitzer color-color diagram are either in region 1 or in the blue clump. In both cases, the radio emission is interpreted as emission from an AGN.

Eleven polarized sources $(27 \%)$ are found in the region of PAH-dominated galaxies, with $\log \left(S_{8.0} / S_{4.5}\right)>-0.5$, a few dex above the blue clump. Their location in the color-color diagram suggests either PAH-dominated galaxies at redshift $0.5-1.5$ or galaxies with faint PAH bands at lower redshift (Sajina et al. 2005). Inspection of the FIRST and SWIRE images of these sources shows that all appear to be elliptical galaxies with a smooth morphology, and some have resolved symmetric radio sources, suggestive of radio lobes. This suggests that the polarized radio emission in these galaxies is associated with AGN activity, while the PAH emission originates from dust at a substantial distance from the AGN, where it is shielded from the hard ultraviolet radiation that would destroy the PAHs. Dust in elliptical galaxies is a common phenomenon (Goudfrooij et al. 1994). It can be produced in the envelopes of cool red giant stars or it can be acquired through a merger with a gas-rich galaxy.

The Stokes $I$ sources with no detectable polarized emission generally occupy the same areas of the color-color diagram as the polarized sources, with two exceptions. First, a significant fraction (15\%) of the Stokes $I$ sources is located in region 2, the area where star-forming, PAH-dominated galaxies are expected. 
These objects are generally the faintest Stokes $I$ radio sources, and none would be detectable in polarization at our sensitivity level. The Stokes $I$ sources in region 2 are likely members of a population of star-forming galaxies that is believed to make up a large fraction of the radio source population below $\sim 1 \mathrm{mJy}$. The second exception is that Table 3 indicates an excess of polarized sources $(27 \%)$ relative to sources with no detected polarization $(14 \%)$ in region $3 \mathrm{~b}$, associated with galaxies having PAH emission. This difference is significant if Poisson errors are assumed. Confirmation will require a more complete sample of polarized radio sources identified with Spitzer galaxies.

\subsection{Nature of the Millijansky Polarized Source Population}

The polarized sources found in the ELAIS N1 field have a median Stokes $I$ flux density of $12 \mathrm{mJy}$. Models of radio source populations fitted to the total radio source counts suggest that most radio sources with $1420 \mathrm{MHz}$ flux density $\gtrsim 1 \mathrm{mJy}$ are steepspectrum radio galaxies whose power is ultimately derived from accretion onto a compact object. However, it is not clear a priori that a faint-polarization-selected sample of radio sources is representative of the entire population. In principle, a highly polarized population of faint radio sources may constitute a significant fraction of a sample of faint polarized sources.

In $\S 3.2$ we presented evidence that faint extragalactic polarized radio sources are on average more highly polarized than bright sources, with a median fractional polarization approximately 3 times higher. From an analysis of sources with Stokes $I>100 \mathrm{mJy}$ in the NVSS, Mesa et al. (2002) also noted that the median fractional polarization of radio sources increases with decreasing flux density, from $1.05 \%$ for Stokes $I>800 \mathrm{mJy}$ to $1.84 \%$ between 100 and $200 \mathrm{mJy}$. From a similar analysis of the NVSS, Tucci et al. (2004) showed that the anticorrelation between flux density and percentage polarization occurs only for steep-spectrum sources. They found that the median percentage polarization for steep-spectrum sources increased from $1.14 \%$ for flux densities greater than $800 \mathrm{mJy}$ to $1.77 \%$ between 100 and $200 \mathrm{mJy}$. Our result extends this to much lower flux densities and indicates a much stronger effect for faint sources, resulting in a median polarization of $4.8 \%$ at Stokes $I=10-30 \mathrm{mJy}$. Analysis of Table 1 shows that these faint polarized emitters are dominated by steep-spectrum sources; all but one of the polarized sources for which a 325-1420 MHz spectral index exists (75\%) has $\alpha<-0.4$.

Polarized sources that can be identified with galaxies in the Spitzer ELAIS N1 deep field are associated with elliptical galaxies. Most of the host galaxies have near-infrared colors typical for dust emission from the vicinity of an AGN, or an old stellar population with no significant dust emission. The majority of the polarized sources is associated with AGN activity for this reason. The remaining 11 polarized sources associated with galaxies having PAH emission are also likely to contain an AGN. Some are clearly resolved double-lobed objects in the FIRST images, and all appear to be elliptical galaxies with a smooth brightness distribution. We conclude that there is no evidence for galaxies in our sample of polarized sources in which the radio emission is powered by star formation. The higher fractional polarization of faint radio sources may be related to a population of radio-quiet AGNs in which fainter radio emission correlates with conditions that favor increased polarization, for example more ordered magnetic fields or less internal Faraday depolarization. High-resolution polarimetry of these objects will provide more insight into their nature.

\section{CONCLUSIONS}

We present sensitive observations of a complete sample of compact polarized radio sources, as part of a deep integration of the ELAIS N1 region made with the Synthesis Telescope at the Dominion Radio Astrophysical Observatory. A total of 83 polarized sources was detected in the 10-field mosaic.

The distribution of fractional polarization of faint polarized sources was investigated with a Monte Carlo analysis that generates synthetic source lists with the same noise statistics and observational selection criteria as the data. Maximum likelihood fits of the synthetic source lists to the data in the $\log I-\log p$ plane yielded a best-fitting Gauss-Hermite function with $\sigma_{\Pi_{0}}=7.0 \% \pm$ $1.0 \%, h_{4}=0.05 \pm 0.05$ for the distribution of intrinsic fractional polarization. The data demonstrate a trend of increasing fractional polarization with decreasing flux density.

Polarized source counts from the ELAIS N1 deep field are presented down to $0.5 \mathrm{mJy}$. We find that the Euclidean-normalized polarized counts remain flat below $1 \mathrm{mJy}$. The distribution of fractional polarization derived from our Monte Carlo analysis is convolved with the total-intensity source counts to produce a prediction of the polarized source counts. The predicted Euclidean-normalized polarized counts are nearly flat to $\sim 2 \mathrm{mJy}$, in good agreement with the data. However, the data at the faintest polarized flux densities suggest a continuing trend of increased polarization fraction with decreasing flux density.

The near-infrared color-color diagram for host galaxies identified with the polarized sources in the ELAIS N1 field shows that most of the host galaxies are ellipticals, or galaxies for which the near-infrared spectrum is dominated by stochastically heated very small grains, presumably from the vicinity of an AGN. Some of the host galaxies appear to have PAH bands in their nearinfrared spectrum, but the morphological resemblance with ellipticals, and the fact that some of these polarized sources are resolved radio galaxies in the FIRST survey, indicates that these objects also harbor AGNs. We suggest that the higher degree of polarization indicates a difference between AGNs observed at a flux density of hundreds of millijanskys, and fainter AGNs.

Observations and research on the DRAO Planck Deep Fields are supported by the Natural Sciences and Engineering Research Council of Canada and the National Research Council Canada. Ev Sheehan of DRAO was instrumental in improving the sensitivity of the DRAO Synthesis Telescope, with the outcome recorded in this paper. We are indebted to him for his skill and dedication to this difficult task. The Dominion Radio Astrophysical Observatory is operated as a National Facility by the National Research Council of Canada.

\section{REFERENCES}

Beck, R., \& Gaensler, B. M. 2004, NewA Rev., 48, 1289

Condon, J. J., Cotton, W. D., Greisen, E. W., Yin, Q. F., Perley, R. A., Taylor, G. B., \& Broderick, J. J. 1998, AJ, 115, 1693

Goudfrooij, P., Hansen, L., Jorgensen, H. E., \& Norgaard-Nielsen, H. U. 1994, A\&AS, 105, 341

Gruppioni, C., Mignoli, M., \& Zamorani, G. 1999, MNRAS, 304, 199
Hopkins, A., Windhorst, R., Cram, L., \& Ekers, R. 2000, Exp. Astron., 10, 419

Hopkins, A. M., Afonso, J., Chan, B., Cram, L. E., Georgakakis, A., \& Mobasher, B. 2003, AJ, 125, 465

Landecker, T. L., et al. 2000, A\&AS, 145, 509

Lonsdale, C. J., et al. 2003, PASP, 115, 897 
Mesa, D., Baccigalupi, C., De Zotti, G., Gregorini, L., Mack, K.-H., Vigotti, M., \& Klein, U. 2002, A\&A, 396, 463

Oliver, S., et. al. 2000, MNRAS, 316, 749

Peacock, J. A. 1983, MNRAS, 202, 615

Rengelink, R. B., Tang, Y., De Bruyn, A. G., Miley, G. K., Bremer, M. N., Röttgering, H. J. A., \& Bremer, M. A. R. 1997, A\&AS, 124, 259

Rice, S. O. 1945, Bell Systems Tech. J., 24, 46

Sajina, A., Lacy, M., \& Scott, D. 2005, ApJ, 621, 256

Schilizzi, R. T. 2004, Proc. SPIE, 5489, 62

Simmons, J. F. L., \& Stewart, B. G. 1985, A\&A, 142, 100

Simpson, C., et al. 2006, MNRAS, 372, 741

Taylor, A. R., et al. 2003, AJ, 125, 3145
Tucci, M., Martínez-González, E., Toffolatti, L., González-Nuevo, J., \& De Zotti, G. 2004, MNRAS, 349, 1267

van der Marel, R. P., \& Franx, M. 1993, ApJ, 407, 525

Vinokur, M. 1965, Ann. d'Astrophys., 28, 412

White, R. L., Becker, R. H., Helfand, D. J., \& Gregg, M. D. 1997, ApJ, 475, 479

Willis, A. G. 1999, A\&AS, 136, 603

Windhorst, R. A. 2003, NewA Rev., 47, 357

Windhorst, R. A., Mathis, D., \& Neuschaefer, L. 1990, in ASP Conf. Ser. 10, Evolution of the Universe of Galaxies, ed. R. G. Kron (San Francisco: ASP), 389 